\title{
Comportamiento dinámico de la conductividad térmica de material cerámico de estructura $\left(\mathrm{Al}_{2} \mathrm{O}_{3}\left(\mathrm{ZnO} / \mathrm{SnO}_{2}\right)+\mathrm{Ti}_{0,1} \mathrm{O}_{2}\right)$
}

\author{
RODRÍGUEZ P. OMAR \\ Universidad Central. Departamento de Ingeniería Electrónica. Facultad de Ingeniería \\ Carrera 5 No. 21 - 38. Bogotá D. C.. Colombia
}

\begin{abstract}
En este trabajo, se presentan los resultados del comportamiento simulado y la medición experimental de la conductividad térmica $\sigma_{\mathrm{T}}$ de material cerámico crudo de fases $\left(\mathrm{Al}_{2} \mathrm{O}_{3}\left(\mathrm{ZnO} / \mathrm{SnO}_{2}\right)+\mathrm{Ti}_{0,1} \mathrm{O}_{2}\right)$, utilizado en la fabricación de sensores de radiación térmica para el control de procesos industriales. Los resultados anteriores se obtuvieron teniendo en cuenta la aplicación de un modelo matemático de radiación de cuerpo negro, como una función no lineal dependiente de: la temperatura, permitividad eléctrica relativa y variables como humedad relativa y voltaje medidos con un dispositivo electrónico desarrollado por el grupo ESSOPTO de la Universidad Central. Por otro lado, se calculó y se simuló el comportamiento térmico del potencial químico responsable de la propagación de la energía en la estructura del compuesto $\left(\mathbf{A l}_{2} \mathbf{O}_{3}\left(\mathrm{ZnO}_{\mathbf{O}}\right.\right.$ $\left.\mathrm{SnO}_{2}\right)+\mathrm{Ti}_{0,1} \mathrm{O}_{2}$ ), dependiendo del tipo de distribución del tipo de portador (n) y (p) del material.
\end{abstract}

Palabras clave: Densidad aparente, conductividad térmica, humedad relativa, permitividad eléctrica, voltaje.

\section{INTRODUCCIÓN}

En el siguiente trabajo, es presentan los resultados del comportamiento simulado y medición experimental de la conductividad térmica $\sigma_{\mathrm{T}}$ para material cerámico crudo de fases $\left(\mathrm{Al}_{2} \mathrm{O}_{3}\left(\mathrm{ZnO} / \mathrm{SnO}_{2}\right)+\mathrm{Ti}_{0,1} \mathrm{O}_{2}\right)$, utilizado en la fabricación de sensores de radiación térmica para procesos industriales. Los resultados experimentales se obtuvieron teniendo en cuenta la aplicación de un modelo matemático de radiación de cuerpo negro como función no lineal dependiente de la temperatura, permitividad eléctrica relativa, humedad relativa HR y voltaje medidos con un dispositivo electrónico diseñado en la Universidad Central por el grupo de investigación en Estado Sólido y Optoelectrónica ESSOPTO. De otro lado, se calculó y se simuló el comportamiento térmico del potencial químico responsable de la propagación de la energía en la estructura del compuesto $\left(\mathrm{Al}_{2} \mathrm{O}_{3}\left(\mathrm{ZnO} / \mathrm{SnO}_{2}\right)+\mathrm{Ti}_{0,1} \mathrm{O}_{2}\right)$, dependiendo de la distribución de los portadores tipo (n) y (p) del material.

\subsection{Planteamiento del problema}

En el proceso de fabricación y caracterización experimental de sensores de radiación térmica utilizados en el control de procesos industriales, tener un medio activo sensible a los cambios térmicos del medio ambiente, implica contar con un protocolo de caracterización experimental de las propiedades termo - mecánicas del compuesto $\left(\mathrm{Al}_{2} \mathrm{O}_{3}(\mathrm{ZnO} /\right.$ $\left.\mathrm{SnO}_{2}\right)+\mathrm{Ti}_{0,1} \mathrm{O}_{2}$ ), que reflejen en sí el comportamiento de la resistencia mecánica, la dilatación térmica y las propiedades de transporte de calor en esta clase de materiales. La mayor dificultad se presenta en la obtención de un modelo teórico que describa el comportamiento del potencial químico como función de la distribución de los portadores de carga tipo $(n / p)$ y que se ajuste a los datos experimentales.

Por la anterior razón, en el laboratorio del grupo ESSOPTO, se diseñó un dispositivo electrónico que determina directamente las corrientes térmicas generadas por la propagación del calor en la muestra, junto con los valores de voltaje que genera la distribución de dipolos en las redes locales del compuesto, que dan cuenta de la influencia de las diferentes estructuras del compuesto en el factor de pérdida de la energía por disipación de calor.

\section{MODELO TEÓRICO}

Si se considera la estructura $\left(\mathrm{Al}_{2} \mathrm{O}_{3}\left(\mathrm{ZnO} / \mathrm{SnO}_{2}\right)+\mathrm{Ti}_{0,1} \mathrm{O}_{2}\right)$, el objeto de estudio del presente problema, como un sistema clásico de $\mathrm{N}$ dipolos, entonces cualquier onda térmica que pueda interactuar con los dipolos locales de la red, generará un sistema de disipación térmica anisotrópica, creando con esto corrientes térmicas sin una dirección preferencial de movimiento de la onda térmica que estará determinadapor el gradiente térmico que la estructura genera. Esta propiedad estructural de propagación y disipación de la energía se conoce como conductividad térmica.

Tanto los resultados simulados como los resultados experimentales del presente informe de investigación, se basaron en el modelo teórico de radiación de cuerpo negro presentado en [12].

$$
\sigma_{T}=\frac{d Q}{d t} \cdot \frac{1}{S \cdot \nabla T}
$$


Donde: Q - calor transferido a la muestra por la fuente; $S$ - área de la muestra expuesta directamente a la fuente; $\nabla T$ gradiente térmico en el interior de la muestra.

El modelo teórico obtenido que describe el comportamiento del potencial químico en función de la distribución de los portadores tipo $(\mathrm{n} / \mathrm{p})$ del material es:

$$
\boldsymbol{\nabla}^{2} V(x)=-\frac{1}{\mu} \operatorname{Cth}\left(\frac{X_{j-x}}{L_{n}}-w t\right)\left[\boldsymbol{w}-\frac{\mu E}{L_{n}}\right]-\frac{D_{n}}{L_{n}^{2} \mu}
$$

Donde: $\mathrm{L}_{\mathrm{n}}$ - longitud de difusión de los portadores en la estructura; $D_{n}$ - coeficiente de difusión de los portadores; $\square$ movilidad de portadores; $X_{j}$ - camino libre medio recorrido de los portadores.

Resolviendo la ecuación (2) y calculando el potencial químico, la relación con la conductividad eléctrica es:

$$
V(x)=\frac{n(x, t)}{C_{v}} \frac{\sigma_{T}}{\sigma_{e}}
$$

Donde: $\mathrm{n}(\mathrm{x}, \mathrm{t})$ - función de distribución de los portadores en la estructura; $C_{v}$ - capacidad calorífica de la muestra; $\sigma_{T}$ - conductividad térmica de la muestra; $\sigma_{\mathrm{e}}$ - conductividad eléctrica de la muestra.

\section{PROCEDIMIENTO EXPERIMENTAL. (TÉCNICA APLI- CADA)}

Para el desarrollo experimental del presente trabajo, se prepararon probetas de material cerámico crudo con diferente concentración, tamaño de grano y grado de compactación. El valor de la RH osciló entre 5 y $12 \%$ por peso de la muestra logrando después una mezcla homogénea muy cercana a la fase plástica del compuesto.Acto seguido, se compactaron las probetas en una prensa mecánica diseñada especialmente para incluir en el volumen de cada probeta cuatro electrodos diametralmente opuestos separados a una distancia de $1.5 \mathrm{~cm}$, con el fin de registrar los valores de voltaje y corriente generados por la acción directa de la fuente externa. Todas las muestras se ubicaron en una cámara en donde la temperatura fue debidamente controlada hasta obtener un valor de secado del $8 \%$ de humedad, luego se procedió a perforar cada una de las probetas para poder acondicionar un sensor de temperatura que determinara la variación interna del calor transferido de la fuente externa a las muestras y viceversa. En el laboratorio de Estado Sólido de la Universidad se acondicionó y organizó un sistema de medición electrónico, (incluyendo un analizador espectral, un osciloscopio y generador de señales) que registrara y analizara el comportamiento eléctrico y térmico de las muestras objetode estudio.

En la última etapa de caracterización, de la corriente, la superficie de las probetas objeto de estudio se dividieron en anillos concéntricos cuyo centro pasó a ser el cero de referencia. Seguidamente se estableció un patrón métricoasignado al sistema coordenado cartesiano para ubicar los límites de las zonas o anillos, después se procedió a medir punto a punto el valor de la corriente sin que a las muestras se les conectara a una fuente de energía.

\section{ANÁLISIS DE RESULTADOS}

En el planteamiento y desarrollo del modelo matemático se encontró que para cualquier distribución de portadores de carga del tipo hiperbólico, el cálculo del potencial químico y por ende la determinación de la conductividad térmica, conducía al establecimiento de una ecuación diferencial de coeficientes variables conocida como ecuación de Ricatti, lo que dio a entender que la distribución del potencial químico local en la estructura tiene la misma configuración hiperbólica. Por otro lado, si se asumía una distribución de portadores del tipo exponencial, la solución del problema era más sencilla y conducía a una distribución del potencial y de la conductividad térmica del tipo inverso con la longitud de difusión $\mathrm{L}_{\mathrm{n}^{\prime}} \mathrm{L}_{\mathrm{p}}$. Lo anterior aplicaba a distancias en el proceso de simulación de $\mathrm{cm}$, es decir, a ver el potencial macroscópicamente.

Los datos medidos de corriente y voltaje en las muestras, arrojaron un comportamiento acorde a una distribución de tipo hiperbólico, lo que corresponde al proceso de simulación planteado en el presente informe.

El proceso de simulación de la ecuación (2) se llevó a cabo con la utilización del programa de cálculo Matlab 7.1.

El comportamiento experimental de la conductividad térmica $\sigma_{\mathrm{T}}$ se presenta en la Figura 1.

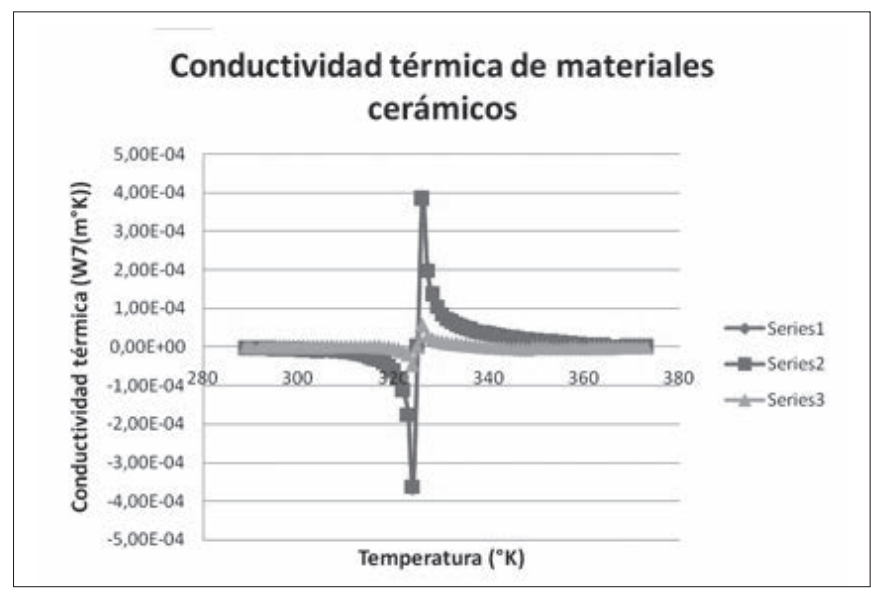

Figura 1: Comparación para tres clases de muestras a diferente temperatura del comportamiento de la conductividad térmica.

El comportamiento dinámico del material en el rango de 0 a $200 \mathrm{~Hz}$, da como resultado inicialmente, la activación de los modos normales de oscilación de los dipolos de la estructura del cercanos a los $21.4 \mathrm{~Hz}$, y posteriormente por efecto de colisión, la activación en la medida del aumento de la frecuencia de la fuente de los dipolos de la red propiamente dicha.

La pendiente negativa de la curva en la figura 2, está asociada a la dirección del vector de polarización $\mathbf{P}$ al interior de la muestra y su magnitud al número de dipolos orientados por campo eléctrico externo y a la forma de oscilación de los mismos.

\subsection{Modelo simulado del potencial químico}

En la figura 2, modelo simulado de la ecuación (2), se muestra la tendencia no lineal del potencial químico en dependencia de la longitud de difusión de los portadores de energía calórica (fonones). Esta forma de distribución genera en ciertas zonas del compuesto una avalancha de portadores de carga, que dependen del gradiente térmico en la muestra, mientras que en otras zonas se genera un represamiento de las mismas producido por el campo eléctrico, sensible 


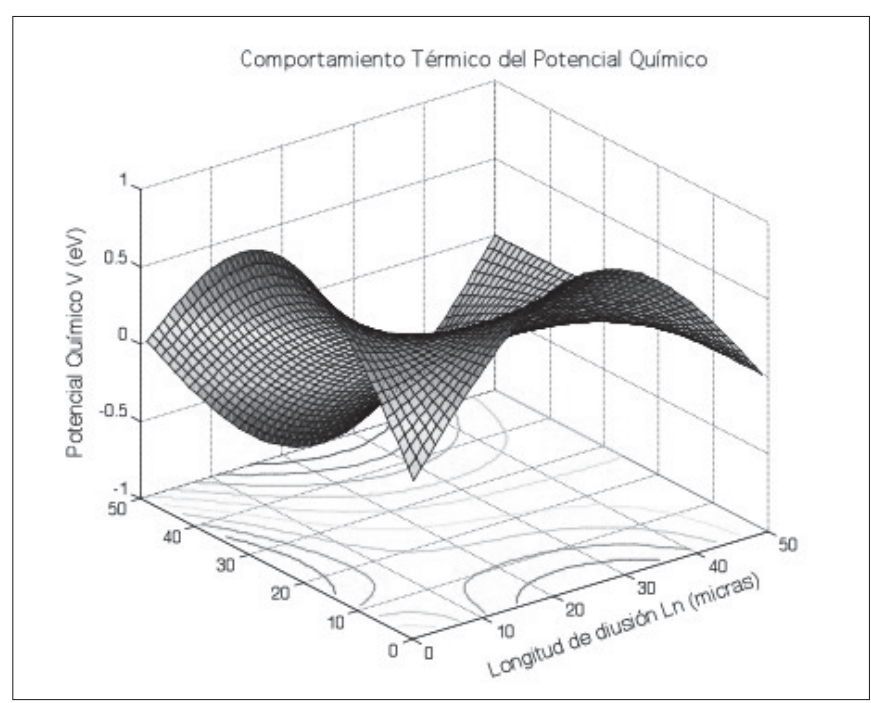

Figura 2: modelo simulado del comportamiento térmico del potencial químico en las muestras cerámicas.

a las variaciones de estructura, y que en su conjunto crean localmente en el compuesto.

\subsection{Discusión de resultados}

Teniendo en cuenta la hipótesis planteada en el marco teórico sobre el comportamiento anisotrópico de las muestras cerámicas, objeto de estudio, la figura 2 demuestra el comportamiento del potencial químico relacionado con la impedancia $Z$ en la dirección (111) de la estructura. Los anteriores resultados están sustentados cada uno con un par de funciones de calibración y los resultados experimentales obtenidos, que fueron escogidas teniendo en cuenta las condiciones iniciales del problema y que satisficieran las ecuaciones dinámicas del comportamiento de la corriente interna en el material.

\section{CONCLUSIONES}

- El comportamiento anisotrópico de la conductividad térmica en las muestras utilizadas en el presente informe, se manifiesta en la figura 1 alrededor de los 325 grados Kelvin, dado que la propagación de la onda térmica en materiales amorfos se hace más efectiva cuando la estructura atómica es altamente desordenada e irregular que en los cerámicos cristalinos.

- Aunque los fonones son los responsables del transporte de la energía calorífica en esta clase de estructuras, pues ellos difunden por imperfecciones anisotrópicas locales, el comportamiento asintótico de la conductividad térmica a bajas temperaturas, en un indicio del grado de porosidad de las muestras.

- La aplicación teórica del modelo matemático propuesto en el presente informe y los resultados experimentales, expresan específicamente la influencia de la humedad relativa de las muestras y la densidad de poros, en el comportamiento de la conductividad térmica y la forma del potencial químico (figura 2), modelo que en otros reportes no se ha encontrado hasta el momento.

\section{REFERENCIAS BIBLIOGRÁFICAS}

1. Y. V.Serdyuk, A.D. Podoltsev, S.M. Gubanski. Numerical Simulations and Experimental Study of Frequency - Dependent Dielectric Properties of Composite Material with Stochastic Structure. IEEE transactions on Dielectrics and Electrical Insulation. Vol. 11, No. 3, June 2004.

2. Y. Du,M. Zahn, N. Altamirano, M. Sarda. Moisture and Temperature Effects on the Dielectric Spectrum of Transformer Pressboard. 2002 Annual Report Conference on Electrical Insulation and Dielectric Phenomena.

3. J.C.Duncany Marsh, R. D. L. Wide Frequency Range Dielectric Spectroscopy (Application to Food Materials). 1995 IEEE $5^{\text {th }}$ International Conference on Conduction and Breakdown in Solid Dielectrics.

4. O, Rodríguez P. Determinación de la influencia del factor estructural en el comportamiento de la permitividad relativa de materias primas cerámicas naturales bajo la acción de un campo eléctrico uniforme. XLIII Congreso de la Sociedad de Cerámica y Vidrio. Manises, 19 - 22 Noviembre de 2003 España.

5. E. Tuncer, Y. V. Serdyuk, y S. M. Gubanski, Dielectric Mixtures. Electrical Properties and Modeling, IEEE Transactions on Dielectrics and Electrical Insulation. Vol. 9 No. 5, October 2002

6. J.H. Duncan, D.G. Haeni, J.H. Lettieri, J. Theis. Novel exchange mechanisms in the surface diffusion of oxides. Letter to the editor. Journal of Physics: Condensed mater. 16 (2004) L187 - L192.

7. O. Rodriguez P.. Calculation of the relative humidity of raw ceramic material dopped with $\left(\mathrm{CuSO}_{4}\left(\mathrm{ZnO} / \mathrm{SnO}_{2}\right)+\mathrm{H}_{2} \mathrm{O}\right)$ using the characterization in frequency. ICC Toronto Canada. Junio 23 - 302006.

8. G. L. Kellogy P. J. Feibelmann. Surface self - diffusion on PT (001) by an atomic exchange mechanism. 1990 Phys. Rev. Lett. 643147

9. R. Tromp, Novel exchange mechanisms in the surface diffusion oxides. 2003 Nat. Mater. 2212

10. G. Henkelman, y H. Jonsson, Surface diffusion atoms go underground. 2001. J. Chem. Phys. 1159657.

11. A. F., D’Souza, Design of control systems. Prentice Hall international 1999.

12. I. V. Saveliev, Física general T3. Ed. Mir, Moscú 1998 